\title{
CULTIVO DE TOMATE (Solanum lycopersicum) HIDROPÔNICO SOB DIFERENTES NÍVEIS DE FÓSFORO E POTÁSSIO EM SOLUÇÃO NUTRITIVA
}

\author{
Nilvan Carvalho Melo' ${ }^{1}$ Luma Castro de Souza ${ }^{1}$; Vicente Filho Alves Silva²; Rafaelle \\ Fazzi Gomes²; Cândido Ferreira de Oliveira Neto³ ${ }^{3}$ Deborah Luciany Pires Costa ${ }^{4}$. \\ ${ }^{1}$ Doutorandos em Ciência do Solo da Universidade Estadual Paulista (UNESP/FCAV). Jaboticabal, São Paulo, \\ Brasil.nilvan.melo@ufra.com.br, lumasouza30@hotmail.com \\ ${ }^{2}$ Doutorandos em Produção Vegetal da Universidade Estadual Paulista (UNESP/FCAV). Jaboticabal, São Paulo, \\ Brasil. vicentedelta@yahoo.com.br, rafaelle.fazzi@yahoo.com.br \\ ${ }^{3}$ Professor Doutor da Universidade Federal Rural da Amazônia (UFRA). Belém, Pará, Brasil. \\ ccandido.neto@ufra.edu.br \\ ${ }^{4}$ Graduanda em Agronomia da Universidade Federal Rural da Amazônia (UFRA). Belém, Pará, Brasil. \\ deborahpires.agro@gmail.com
}

\begin{abstract}
RESUMO: O tomate está entre as hortaliças mais consumidas no mundo, sua produção anual no Brasil é estimada em torno de quatro milhões de toneladas. O objetivo do trabalho foi estudar o efeito de diferentes doses de fósforo e potássio no crescimento de tomateiro, cultivado em sistema hidropônico. O trabalho foi conduzido em casa de vegetação do Instituto de Ciências Agrárias da Universidade Federal Rural da Amazônia, no período de outubro de 2008 a janeiro de 2009, em solução nutritiva. O delineamento experimental utilizado foi inteiramente casualizado em esquema fatorial $3 \times 3$ (três doses de fosfóro e três doses de potássio) com quatro repetições. Cada parcela foi constituída de uma planta por vaso. Não houve interação significativa para os fatores avaliados. Os tratamentos constituídos pela combinação P1K2 e P2K2 proporcionaram melhor desempenho para altura de planta. Para massa fresca da parte aérea e massa fresca de raiz, $\mathrm{P} 2 \mathrm{~K} 1$ proporcionou melhor incremento em relação às demais doses. Na massa seca total acumulada, os tratamentos P1K1 e P2K1 proporcionaram maiores incrementos, 62,7 e 71,9 $\mathrm{g}$ planta $^{-1}$ respectivamente. Com base nisso, o tratamento mais eficiente para a produção do tomateiro foi o $\mathrm{P} 2 \mathrm{~K} 1$, que promoveu maior crescimento em todos os parâmetros avaliados.
\end{abstract}

PALAVRAS-CHAVE: Cultivo sem solo. Fertilizantes. Solanum lycopersicum.

\section{CULTURE OF TOMATO (Solanum lycopersicum) HYDROPONIC UNDER DIFFERENT LEVELS OF PHOSPHORUS AND POTASSIUM NUTRIENT SOLUTION}

\begin{abstract}
Tomatoes are among the most consumed vegetables in the world, its annual production in Brazil is estimated at around four million tonnes. The objective was to study the effect of different doses of phosphorus and potassium on the growth of tomato grown hydroponically. The work was conducted in the greenhouse of the Institute of Agricultural Sciences, Federal Rural University of Amazonia, from October 2008 to January 2009, in nutrient solution. The experimental design was completely randomized factorial $3 \times 3$ (three doses of phosphorus and potassium three doses) with four replications. Each plot consisted of one plant per pot. There was no significant interaction for the factors evaluated. The treatments consisted of the combination P1K2 and P2K2 in better performance for plant height. For fresh weight of shoot and root fresh weight, P2K1 provided better growth compared to the other doses. In the total dry mass accumulation, the P1K1 and P2K1
\end{abstract}


treatments provided greater increments, 62.7 and $71.9 \mathrm{~g} \mathrm{plant}^{-1}$ respectively. Based on this, the most effective treatment for the production of tomato was the P2K1, which promoted greater growth in all parameters evaluated.

KEYWORDS: Soilless cultivation. Fertilizers. Solanum lycopersicum.

\section{CULTURA DE TOMATE (Solanum lycopersicum) HIDROPÓNICA BAJO DIFERENTES NIVELES DE FÓSFORO Y POTASIO EN SOLUCIÓN NUTRITIVA}

RESUMEN: El tomate es uno de los vegetales más consumidos en el mundo, su producción anual en Brasil se estima en alrededor de cuatro millones de toneladas. El objetivo fue estudiar el efecto de diferentes dosis de fósforo y potasio en el crecimiento de plantas de tomate cultivadas hidropónicos. El trabajo se realizó en el invernadero del Instituto de Ciencias Agrícolas de la Universidad Federal Rural de la Amazonia, entre octubre de 2008 enero de 2009, en la solución nutritiva. El diseño experimental fue completamente al azar en el factorial 3 x 3 (tres dosis de fósforo y potasio tres dosis) con cuatro repeticiones. Cada parcela constaba de una planta por maceta. No hay interacción significativa de los factores evaluados. Los tratamientos consisten en una combinación P1K2 P2K2 y dieron como resultado un mejor rendimiento para la altura de la planta. Para peso fresco de brote y raíz peso fresco, P2K1 proporcionó mejor crecimiento en comparación con las otras dosis. En la acumulación total de masa seca, y los tratamientos P2K1 P1K1 ofrecido mayores incrementos de 62.7 y 71.9 g planta $^{-1}$, respectivamente. Basado en esto, el tratamiento más eficaz para la producción de tomate fue la $\mathrm{P} 2 \mathrm{~K} 1$, que promovió mayor crecimiento en todos los parámetros evaluados.

PALABRAS-CLAVE: Cultivo sin suelo. Fertilizantes. Solanum lycopersicum.

\section{INTRODUÇÃO}

O tomate está entre as hortaliças mais consumidas no mundo, sendo uma fonte de vitaminas $\mathrm{A}$ e $\mathrm{C}$ e de sais minerais como potássio e magnésio. É um fruto originário dos países andinos. Pertence à família das Solanáceas. A produção anual brasileira de tomate é estimada em 4,4 milhões de toneladas, sendo dois milhões de toneladas (77\% do total) destinados ao mercado in natura e o restante ao processamento industrial da polpa (FAO, 2013).
As técnicas de cultivo protegido da cultura do tomate minimizam as perdas provocadas pelas diferentes condições do clima e do ambiente, além de otimizar a utilização dos insumos. O cultivo da cultura do tomate em lugares protegidos no período da entressafra pode pode aumentar a produtividade, sendo esses valores maiores que $100 \mathrm{t} \mathrm{ha}^{-1}$ em ciclos de 150 dias (ANDRIOLO et al., 1997). Além disso, o aumento na produtividade do tomateiro produzido por meio de cultivo hidropônico tem sido de 20\%-25\% sobre o 
obtido pelo cultivo em solo, devido diferentes fatores, ou em algumas situações devido ao solo pobre em nutriente ou até mesmo devido à ocorrência de de pragas na lavoura do tomate (MARTINEZ; BRACINI, M.; BRACINI, A. et al., 1997). E dentre as técnicas de cultivo protegido a hidroponia se destaca, sendo esta definida como um cultivo sem solo, no qual o sistema radicular recebem uma solução nutritiva contendo os nutrientes necessários para o seu desenvolvimento (HEBBAR et al., 2004).

As vantagens do cultivo hidropônico em relação ao cultivo com o uso do solo são: maior eficiência na regulação da nutrição das plantas, possibilita o emprego em diferentes regiões do planeta com deficiência de terras adequadas para o cultivo, o uso mais eficiente e adequado da água e dos fertilizantes e maior densidade de plantio, o que resulta em maior produtividade (MARTINEZ; BRACINI, M.; BRACINI, A. et al., 1997).

Dados na literatura mostram que o tomateiro é uma das plantas mais exigentes em adubação, e conforme a etapa de desenvolvimento, a cultivar, a temperatura, o solo, a luminosidade, a umidade relativa e o manejo adotado, os teores médios de nutrientes no tecido vegetal podem variar (ALVARENGA, 2004). É sabido que a qualidade, bem como a produtividade são influenciados pela nutrição mineral adequada, uma falta ou excesso dos nutrientes compromete o metabolismo das plantas, direta ou indiretamente, contribuindo para modificações na forma e composição química, morfologia e anatomia (SASAKI; SENO, 1994; MARSCHNER, 1995; ALVARENGA, 2004).

Dentre os macronutrientes o potássio se torna de fundamental importância na cultura do tomateiro, devido estar ligado aos processos de síntese e translocação de carboidratos para os frutos, assim alterações nos níveis de potássio fornecidos as plantas podem influenciar na qualidade dos frutos (PIMENTEL, 2004).

Outro macronutriente que assume grande importância é o fósforo, um dos macronutrientes que mais limita a produção das culturas no Brasil. Uma de suas principais funções está relacionada ao aspecto estrutural e também no processo de transferência / armazenamento de energia (PRADO, 2008).

Com base no que foi exposto o presente trabalho teve por objetivo estudar o efeito de diferentes doses de fósforo e potássio no crescimento de tomateiro, cultivado em sistema hidropônico.

\section{MATERIAL E MÉTODOS}

O trabalho foi conduzido em casa de vegetação do Instituto de Ciências Agrárias 
da Universidade Federal Rural da Amazônia, no período de outubro de 2008 a janeiro de 2009.

$\mathrm{O}$ delineamento experimental usado foi inteiramente casualizado em esquema fatorial $3 \times 3$ (três doses de fósforo e três doses de potássio), com quatro repetições, perfazendo a seguinte combinação: $\mathrm{P}_{0} \mathrm{~K}_{0}$, $\mathrm{P}_{0} \mathrm{~K}_{1}, \mathrm{P}_{0} \mathrm{~K}_{2}, \mathrm{P}_{1} \mathrm{~K}_{0}, \mathrm{P}_{1} \mathrm{~K}_{1}, \mathrm{P}_{1} \mathrm{~K}_{2}, \mathrm{P}_{2} \mathrm{~K}_{0}, \mathrm{P}_{2} \mathrm{~K}_{1}$, $\mathrm{P}_{2} \mathrm{~K}_{2}$. Cada parcela experimental foi constituída por uma planta por vaso com capacidade para $5 \mathrm{~kg}$ de sílica moída.

As mudas foram produzidas em bandejas de poliestireno expandido, com 128 células. Essas mudas foram formadas de sementes da cultivar C-38-D Novo fornecidas pela Embrapa Amazônia Oriental. Foram semeadas duas sementes por célula, sendo o transplantio realizado 20 dias após a germinação, quando as mesmas apresentavam de três a cinco folhas definitivas. A partir desse periodo forneceu-se solução completa diluída 5 vezes $(1: 5)$.

A solução nutritiva utilizada seguiu a recomendação de Hoagland e Arnon (1950) modificada para níveis de P e K. Foram realizados oito tratamentos com diferentes níveis, conforme os níveis dos nutrientes apresentados a seguir (Tabela 1).

Tabela 1 - Niveis e concentrações de fósforo e potássio utilizados nas combinações dos tratamentos.

\begin{tabular}{ccc}
\hline Níveis & $\mathrm{P}$ & $\mathrm{K}$ \\
\hline 0 & $0 \mathrm{ppm}$ & $0 \mathrm{ppm}$ \\
1 & $97 \mathrm{ppm}$ & $234 \mathrm{ppm}$ \\
2 & $194 \mathrm{ppm}$ & $468 \mathrm{ppm}$ \\
\hline
\end{tabular}

A colheita das plantas iniciou-se a partir de 45 dias do início dos sintomas visuais como, folhas mais velhas arroxeadas (fosfóro), e clorose marginal das folhas mais velhas (potássio) nos devidos tratamentos (PRADO, 2008).

Foram avaliadas as seguintes caracteristicas: massa fresca e seca da parte aerea; massa fresca e seca de raiz; e altura de plantas.
Para as avaliações, as plantas foram separadas em parte aérea e raízes, pesadas, em seguida lavadas em água destilada, e colocadas para secar em estufa a $65^{\circ} \mathrm{C}$ até massa constante.

Após a obtenção dos dados, os mesmo foram submetidos à análise de variância, e a comparação de médias foi feita por meio de Tukey a 5\% de probabilidade, usando o software SISVAR (FERREIRA, 2011). 
RESULTADOS E DISCUSSÃO

De acordo com a análise de variância, não houve interação significativa para os fatores avaliados, sendo os mesmo discutidos separadamente.
Para a caracteristica altura de plantas (Gráfico 1) os melhores desempenhos foram obtidos nas combinações de P1K2 e P2K2, diferindo estatisticamente dos demais tratamento.

Gráfico 1 - Altura de plantas de tomate cultivadas sob diferentes níveis de P e K em solução nutritiva. UFRA, Belém-PA.

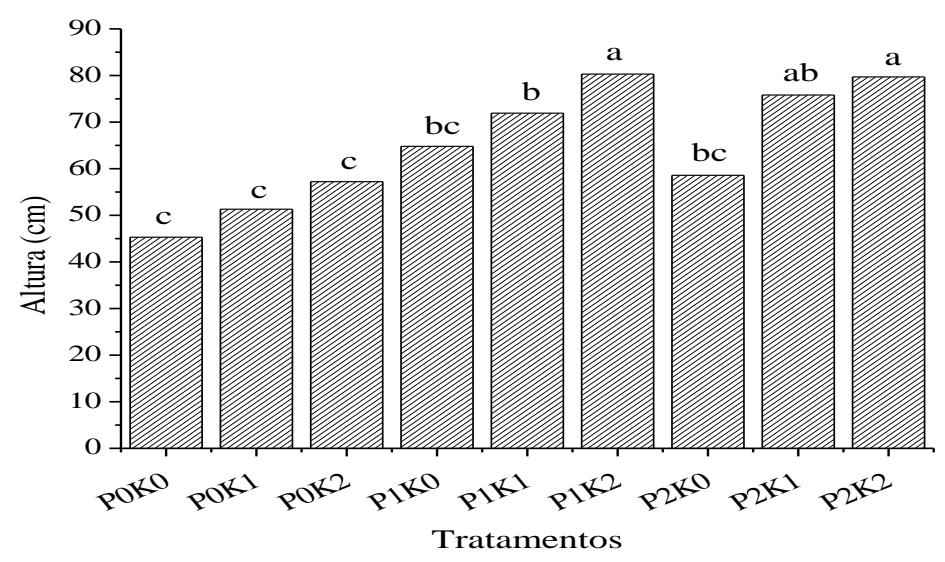

NOTA: Letras distintas indicam diferença significativa pelo teste de Tukey $(p<0,05)$.

Este resultado demonstra a importância desses dois nutrientes, fosfóro e potássio no desenvolvimento e crescimento da planta, haja vista que o tomateiro é uma das hortaliças mais exigentes em nutrientes, respondendo ao uso de doses elevadas (FONTES; SILVA, 2002).

Para massa fresca da parte aérea (Tabela 2) é possivel observar melhor comportamento para os tratamentos $\mathrm{P} 1 \mathrm{~K} 1$, P1K2 e P2K1, diferindo dos demais tratamentos. Enquanto, na massa fresca de raiz (Tabela 2) o tratamento P2K1 apresentou maior valor $\left(28,5 \mathrm{~g}_{\text {planta }}{ }^{-1}\right)$, diferindo dos demais tratamentos. Esse aumento nos teores de fosfóro e potássio aplicados evidenciam o efeito desses nutrientes no desenvolvimento de caracteristicas vegetativas e reprodutivas das plantas (MARSCHNER, 1995). Plantas com teores adequados de potássio, por exemplo, tem o número e tamanho dos estômatos por unidade de área foliar 
aumentados, facilitando as trocas gasosas (MORAES, 2006), podendo com isso aumentar a fotossintese, e consequentemente a massa fresca das plantas.

A massa seca total acumulada pelas plantas (Tabela 2) apresentou maiores valores nos tratamentos com aplicação de P1K1 (62,7 g planta $\left.^{-1}\right)$ e P2K1 (71,9 g planta $\left.{ }^{-1}\right)$.
Assim o potássio devido aos seus diversos papéis na planta, proporciona efeitos diretos na produção da maioria das culturas, e juntamente com o fósforo, proporciona nas plantas sob deficiência mecanismo de adaptação, tendo maior translocação de carboidratos para a raiz, minimizando os prejuízos ao sistema radicular (PRADO, 2008).

Tabela 2 - Massa fresca da parte aérea, massa fresca de raiz, massa seca total e relação parte aérea/raiz, de plantas de tomate submetidas a diferentes níveis de P e K em solução nutritiva. UFRA, Belém-PA.

\begin{tabular}{ccccc}
\hline Tratamentos & MFPA & MFR & MST & PA/Raiz \\
\cline { 2 - 4 } & & g planta $^{-1}$ & \\
\hline P0K0 & $15,6 \mathrm{c}$ & $12,5 \mathrm{c}$ & $28,1 \mathrm{c}$ & 1,248 \\
P0K1 & $20,2 \mathrm{bc}$ & $13,6 \mathrm{c}$ & $33,8 \mathrm{c}$ & 1,485 \\
P0K2 & $25,4 \mathrm{bc}$ & $9,5 \mathrm{c}$ & $34,9 \mathrm{c}$ & 2,674 \\
P1K0 & $18,1 \mathrm{c}$ & $15,5 \mathrm{bc}$ & $33,6 \mathrm{c}$ & 1,168 \\
P1K1 & $39,9 \mathrm{a}$ & $22,8 \mathrm{~b}$ & $62,7 \mathrm{a}$ & 1,750 \\
P1K2 & $36,1 \mathrm{a}$ & $25,9 \mathrm{ab}$ & $62,0 \mathrm{ab}$ & 1,394 \\
P2K0 & $28,6 \mathrm{~b}$ & $11,2 \mathrm{c}$ & $39,8 \mathrm{bc}$ & 2,554 \\
P2K1 & $43,4 \mathrm{a}$ & $28,5 \mathrm{a}$ & $71,9 \mathrm{a}$ & 1,523 \\
P2K2 & $35,8 \mathrm{ab}$ & $24,8 \mathrm{ab}$ & $60,6 \mathrm{ab}$ & 1,444 \\
\hline
\end{tabular}

NOTA: Médias seguidas da mesma letra, na coluna, não diferem entre si, pelo teste de Tukey a $5 \%$ de probabilidade.

\section{CONCLUSÕES}

O tratamento mais eficiente para a produção do tomateiro foi o P2K1, que promoveu maior crescimento em todos os parâmetros avaliados.

\section{REFERÊNCIAS}

ALVARENGA, M. A. R. Tomate: produção em campo, em casa de vegetação e em hidroponia. Viçosa: Ed. da UFV, 2004.

ANDRIOLO, J. L.; DUARTE, T. S.; LUDKE, L.; SKREBSKY, E. C. Crescimento e desenvolvimento do tomateiro cultivado em substrato com fertirrigação. Horticultura Brasileira, v. 15, p. 28-32, Dec. 1997.

FERREIRA， D. F. Sisvar: a computer statistical analysis system. Ciência e Agrotecnologia, v. 35, n. 6, p. 1039-1042, nov./dez. 2011. 
FONTES, P. C. R.; SILVA, D. J. H. Doenças e pragas: é seguro comer tomate?. In: _. Produção de Tomate de Mesa, p. $97-129,2002$.

FOOD AND AGRICULTURAL

Statistical Yearbook 2013. Disponível em: $\quad$ http://faostat.fao.org/ site/339/default.aspx. Acesso em: 05 abr. 2013.

HEBBAR, S. S. ; RAMACHANDRAPPA, B. K. ; NANJAPPA, H. V. ; PRABHAKAR, M. Studies on NPK drip fertigation in field grown tomato (Lycopersicon esculentum Mill.). European Journal of Agronomy, v. 21, n. 1, p. 117-127, June, 2004.

HOAGLAND, D. R; ARNON, D. I. The Water culture method for growing plants without soil. Berkeley: Univ. of California, 1950. (California Agricultural Experiment Station, Circular, 347).

MARSCHNER, H. Mineral nutrition of higher plants. $2^{\text {nd }}$ ed. New York: Academic Press, 1995.

MARTINEZ, H. E. P.; BRACCINI, M. C. L.; BRACCINI, A. L. Cultivo hidropônico do tomateiro (Lycopersicon esculentum Mill.). Revista UNIMAR, v. 19, n. 3, p. 721-740, 1997.

MORAES, I. V. M. Cultivo de hortaliças: dossiê técnico. Rio de Janeiro: Rede de tecnologia do Rio de Janeiro, 2006.

PIMENTEL, C. A. A relação da planta com a água. Seropédica: ENA, 2004.

PRADO, R. M. Nutrição de plantas. São Paulo: Ed. da Unesp, 2008.

SASAKI, J. L. S.; SENO, S. Importância da adubação na qualidade de algumas olerícolas (alho, cebola, couve flor, pimentão e tomate). In: SÁ, E.; BUZZTI,
S. Importância da adubação na qualidade dos produtos agrícolas. São Paulo: Ícone, 1994. p. 331-343. 